\title{
The Neural Mechanisms Underlying the Influence of Pavlovian Cues on Human Decision Making
}

\author{
Signe Bray, ${ }^{1}$ Antonio Rangel, ${ }^{1,3}$ Shinsuke Shimojo, ${ }^{1,2}$ Bernard Balleine, ${ }^{4}$ and John P 0’Doherty ${ }^{1,3}$ \\ ${ }^{1}$ Computation and Neural Systems, ${ }^{2}$ Division of Biology, and ${ }^{3}$ Division of Humanities and Social Sciences, California Institute of Technology, Pasadena, \\ California 91125, and ${ }^{4}$ Department of Psychology and the Brain Science Institute, University of California Los Angeles, Los Angeles, California 90024
}

In outcome-specific transfer, pavlovian cues that are predictive of specific outcomes bias action choice toward actions associated with those outcomes. This transfer occurs despite no explicit training of the instrumental actions in the presence of pavlovian cues. The neural substrates of this effect in humans are unknown. To address this, we scanned 23 human subjects with functional magnetic resonance imaging while they made choices between different liquid food rewards in the presence of pavlovian cues previously associated with one of these outcomes. We found behavioral evidence of outcome-specific transfer effects in our subjects, as well as differential blood oxygenation level-dependent activity in a region of ventrolateral putamen when subjects chose, respectively, actions consistent and inconsistent with the pavlovian-predicted outcome. Our results suggest that choosing an action incompatible with a pavlovian-predicted outcome might require the inhibition of feasible but nonselected action- outcome associations. The results of this study are relevant for understanding how marketing actions can affect consumer choice behavior as well as for how environmental cues can influence drugseeking behavior in addiction.

Key words: instrumental conditioning; decision; fMRI; human; learning; pavlovian conditioning; reward

\section{Introduction}

It is well known that pavlovian cues associated with rewarding outcomes can exert biasing effects on action selection (Colwill and Rescorla, 1988; Balleine, 1994). A form of this effect relevant for decision making is outcome-specific transfer (Rescorla, 1994; Corbit et al., 2001; Holland, 2004; Corbit and Balleine, 2005; Corbit and Janak, 2007). In outcome-specific transfer, an animal's choice between multiple simultaneously available instrumental responses leading to different outcomes can be biased by the presentation of a pavlovian cue previously associated with one of those outcomes, such that the animal will tend to favor the instrumental action corresponding to the particular outcome with which that cue has been associated. Outcome-specific transfer effects are evident, for example, in the impact that in-store advertisements and other marketing strategies have on consumer behavior (Smeets and Barnes-Holmes, 2003), as well as in addictive behavior (Hogarth et al., 2007).

Lesion studies in rodents indicate that the following structures are necessary for outcome-specific transfer to occur: the striatum, including the nucleus accumbens shell (Corbit et al., 2001) and the dorsolateral striatum (Corbit and Janak, 2007), and structures afferent to these regions, including the mediolateral orbito-

Received Feb. 28, 2008; revised April 17, 2008; accepted May 5, 2008.

This work was supported by a Searle scholarship and grants from the National Science Foundation (NSF061714) (J.0.D.), the Gordon and Betty Moore Foundation (J.O.D., A.R.), and the Caltech Brain Imaging Center and the Japan Science and Technology Agency (ERATO) (S.S.). We are grateful to Vivian Valentin for assistance and to Hackjin Kim, Sam Huang, and Shawn Wagner for developing the coil we used to detect swallowing movement.

Correspondence should be addressed to John P O'Doherty, Division of the Humanities and Social Sciences, California Institute of Technology, MC 228-77, Pasadena, CA 91125. E-mail: jdoherty@hss.caltech.edu.

DOI:10.1523/JNEUROSCI.0897-08.2008

Copyright $\odot 2008$ Society for Neuroscience $\quad$ 0270-6474/08/285861-06\$15.00/0 frontal cortex (Ostlund and Balleine, 2007) and basolateral amygdala (Corbit and Balleine, 2005).

Outcome-specific transfer can be differentiated from another form of pavlovian-instrumental interaction called general transfer, in which a pavlovian cue exerts a nonspecific energizing effect on instrumental behavior by increasing the vigor of instrumental responses (Holland, 2004; Corbit and Balleine, 2005). General transfer seems to depend on circuitry involving the ventral striatum and amygdala that is clearly dissociable from that involved in the outcome-specific transfer effect: lesions of the nucleus accumbens core and amygdala central nucleus affect general transfer but leave specific transfer intact, whereas lesions in the nucleus accumbens shell and basolateral amygdala have the converse effect (Corbit et al., 2001; Corbit and Balleine, 2005). In humans, a recent functional magnetic resonance imaging (fMRI) study has implicated human nucleus accumbens and amygdala in general transfer (Talmi et al., 2008), but the brain systems underlying outcome-specific transfer in the human or primate brain more generally have yet to be identified. Furthermore, whereas rodent lesion studies have identified regions that appear to be necessary for specific transfer, the precise functional contribution of each of these regions to this process has yet to be characterized.

The aim of the present study was twofold: first, to determine the neural substrates of the outcome-specific transfer effect in the human brain, and, second, to gain insight into the neural computations within these regions that might underlie this function. To address these aims, we used event-related fMRI to measure blood oxygenation level-dependent (BOLD) responses in human subjects while they made instrumental choices in the presence of pavlovian cues that were either associated with the liquid food reward outcomes generated by some of the actions, or associated 
with an affectively neutral (control) outcome. On the basis of the animal studies, we focused our analysis on the striatum, particularly its ventral aspect, including the nucleus accumbens and adjacent ventral putamen. We also tested for outcome-specific transfer effects in the amygdala.

\section{Materials and Methods \\ Subjects}

Twenty-three healthy, right-handed subjects participated in this study (six females), ranging in age from 18 to 40 (mean $24 \pm 5.3 \mathrm{SD}$ ). One additional subject did not complete the study and was not included in the analysis. All subjects gave informed consent and the study was approved by the Caltech Institutional Review Board.

\section{Stimuli}

Visual stimuli were presented via a projector positioned at the back of the room. Subjects viewed a reflection of the projected image $(800 \times 600$ pixels) in a mirror attached to the scanner head coil. The food rewards were delivered by means of four separate electronic syringe pumps (one for each liquid) positioned in the scanner control room. For each rewarded trial, these pumps pushed $0.6 \mathrm{ml}$ of liquid to the subject's mouth via $\sim 10 \mathrm{~m}$ long polyethylene plastic tubes, the other end of which were held between the subject's lips like a straw while they lay supine in the scanner. Stimulus presentation and response recording were controlled with the Cogent 2000 Matlab (Mathworks) toolbox.

\section{Behavioral procedures}

During both the pavlovian and instrumental training subjects were explicitly asked to learn the cue-outcome and action-outcome relationships. All four training and test sessions described below were performed in the scanner.

Pavlovian training. Pavlovian training consisted of the presentation of associations between simple geometrical visual stimuli (Fig. 1a) and one of four liquid outcomes, three of which were rewarding [chocolate milk (Hershey's, distributed by Dean National Brand Group), cola (CocaCola), and orange juice (Trader Joe's)] and an affectively neutral tasteless control solution, which consisted of the main ionic components of human saliva (25 mm KCl and $2.5 \mathrm{~mm} \mathrm{NaHCO} 3$ ) (Fig. 1a). Cues were presented at the center of the screen for $1.75 \mathrm{~s}$, and then $3 \mathrm{~s}$ after cue offset rewards were delivered with a probability of $50 \%$. The intertrial interval varied uniformly between 1 and $5 \mathrm{~s}$.

Instrumental training. During instrumental training trials, subjects were asked to choose between two button-press actions. Four gray squares at the bottom of the screen corresponded to the four buttons on a response box (Current Designs) that the subjects held in their right hand. Specific actions were made available for selection when the corresponding squares changed color from gray to brown, two at a time. As in the pavlovian trials, the response cues appeared for $1.75 \mathrm{~s}$. Subjects were asked to make a choice during this time. The choice was followed by a $3 \mathrm{~s}$ delay before the outcome associated with the chosen action was delivered on $50 \%$ of trials (Fig. 1 b). The intertrial interval varied uniformly between 1 and $5 \mathrm{~s}$. Responses on each button earned distinct outcomes: two of the buttons led to rewarding outcomes (for example, orange juice and chocolate milk), and two led to the neutral outcome. Therefore, during pavlovian training, subjects experienced four different outcomes, whereas in the instrumental trials they experienced only three.

Training schedule. The first training session consisted entirely of pavlovian trials, 10 of each type for a total of 40 trials and a duration of $\sim 6$ min (Table 1). The second training session consisted entirely of instrumental trials, six of each type for a total of 36 trials and a duration of $\sim 5$ min. In the first two sessions, pavlovian and instrumental trials were presented separately to enhance learning of the respective associations. In the third session, pavlovian and instrumental trials were randomly intermixed, $60(15 \times 4)$ pavlovian trials and $60(10 \times 6)$ instrumental trials, for a duration of $\sim 18 \mathrm{~min}$. Before training and after each session, subjects rated the pleasantness of the stimuli as described below.

Outcome-specific transfer. After the three training sessions subjects performed a series of transfer trials. During transfer trials one of the pavlovian cues was presented simultaneously with the instrumental cues (Fig.
Table 1. Trial composition for training and transfer sessions

\begin{tabular}{|c|c|c|c|c|c|c|}
\hline \multirow[b]{2}{*}{ Phase } & \multirow{2}{*}{$\begin{array}{l}\text { Number of } \\
\text { presentations }\end{array}$} & \multicolumn{3}{|l|}{ Cues } & \multirow{2}{*}{\multicolumn{2}{|c|}{ Outcome }} \\
\hline & & Pavlovian & Instr & & & \\
\hline \multirow[t]{4}{*}{1} & 10 & S1 & & & 01 & \\
\hline & & S2 & & & 02 & \\
\hline & & S3 & & & 03 & \\
\hline & & S4 & & & ON & \\
\hline \multirow[t]{6}{*}{2} & 6 & & R1 & R2 & 01 & 02 \\
\hline & & & R1 & R3 & 01 & ON \\
\hline & & & R1 & R4 & 01 & ON \\
\hline & & & R2 & R3 & 02 & ON \\
\hline & & & R2 & R4 & 02 & ON \\
\hline & & & R3 & R4 & ON & ON \\
\hline \multirow[t]{10}{*}{3} & 10 & S1 & & & 01 & \\
\hline & & S2 & & & 02 & \\
\hline & & S3 & & & 03 & \\
\hline & & S4 & & & ON & \\
\hline & 15 & & R1 & R2 & 01 & 02 \\
\hline & & & R1 & R3 & 01 & ON \\
\hline & & & R1 & R4 & 01 & ON \\
\hline & & & R2 & R3 & 02 & ON \\
\hline & & & R2 & R4 & 02 & ON \\
\hline & & & R3 & R4 & ON & ON \\
\hline \multirow[t]{5}{*}{4} & 25 & S1 & R1 & R2 & & \\
\hline & & S2 & R1 & R2 & & \\
\hline & & S3 & R1 & R2 & & \\
\hline & & S4 & R1 & R2 & & \\
\hline & & S4 & R3 & R4 & & \\
\hline
\end{tabular}

S1-S4, Visual cues; R1-R4, four button response actions; 01-03, liquid rewards; ON, affectively neutral, tasteless control solution.

1c), and as in instrumental training, subjects were asked to choose between two available options. This phase was conducted in extinction, meaning that no outcomes were delivered. The reason for performing this phase in extinction was to allow assessment of the influence of the pavlovian cues on instrumental responding without the confounding effects of the outcomes themselves. Testing for outcome-specific effects in extinction is standard in animal learning studies of this phenomenon (Rescorla, 1994; Blundell et al., 2001; Corbit et al., 2001).

There were five different types of trials. Two of the trial types were designed to test for outcome-specific transfer effects. On these trials, subjects chose between actions associated with two particular reward outcomes, $\mathrm{O} 1$ and $\mathrm{O} 2$ (for example, orange juice and chocolate milk), whereas the concurrently presented pavlovian cue was associated with one of these specific outcomes. One of the specific trial types involved the pavlovian cue paired with outcome O1, and the other specific trial type involved the pavlovian cue paired with $\mathrm{O} 2$. Evidence for an outcomespecific transfer effect would be seen if the presence of the pavlovian cue biased choice toward the action associated with the same outcome as the pavlovian cue. In the subsequent analysis, we pooled over both of the specific trial types, but differentiated between trials in which subjects made choices compatible with the pavlovian outcome from those trials in which subjects made choices that were not compatible.

In a third "pavlovian reward control" trial type, subjects were again presented with the choice between two reward outcomes (O1 and O2), but instead the pavlovian cue was previously associated with a different outcome (for example, cola), that was not compatible with either response option.

In the fourth "pavlovian neutral control" trial type, subjects were again presented with the choice between two reward outcomes (O1 and O2), but the pavlovian cue presented this time was that associated with the affectively neutral outcome.

In the final "neutral choice control" trial type, subjects made choices between actions associated with the affectively neutral outcome, in the presence of a pavlovian cue also associated with a neutral outcome. This last trial type was intended to be a baseline condition for choosing between two options in the presence of a visual cue but in the absence of predicted rewards. Each type of trial was presented 25 times, for a total of 125 trials and a duration of $\sim 20 \mathrm{~min}$. 
a

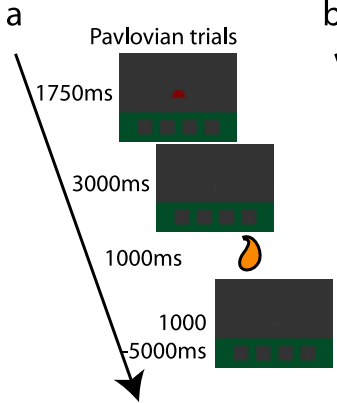

b

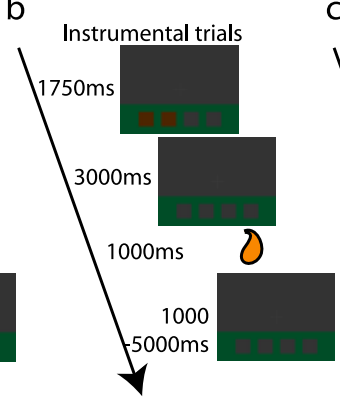

C

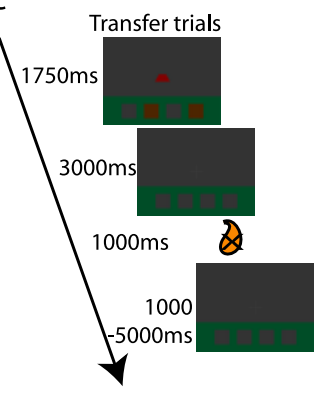

Figure 1. Illustration of the three different trial types in this study. $\boldsymbol{a}$, Pavlovian trial. A visual shape stimulus was presented at the center of the screen for $1.75 \mathrm{~s}$ followed by a fixation cross for $3 \mathrm{~s}$. The liquid outcome corresponding to the stimulus was then delivered with a probability of $50 \%$. One second was allotted for consumption, and the interval between trials varied uniformly between 1 and $5 \mathrm{~s} . \boldsymbol{b}$, Instrumental trial. Two of the four squares at the bottom of the screen changed color from gray to brown for $1.75 \mathrm{~s}$ during which time subjects were instructed to push one of the buttons. The liquid outcome corresponding to their response was delivered after $3 \mathrm{~s}$, with a probability of $50 \%$. One second was allotted for consumption, and the interval between trials varied uniformly between 1 and 5 s. c, Transfer trial. A visual shape stimulus was presented simultaneously with two squares changing color. Subjects were instructed to press one of the corresponding buttons. Timing was similar to the pavlovian and instrumental trials; however, no outcomes were delivered during these trials.

\section{Behavioral measures}

Reaction times. Reaction times to choices were recorded both during the learning trials and the transfer test trials; these can be used as an online measure of learning (O’Doherty et al., 2006).

Pupillary dilation. Pupil diameter was continuously measured during scanning using an Applied Science Laboratories MRI-compatible eyetracking system. Pupil reflex amplitude has been shown to be modulated by arousal level and can thus be used as an index of conditioning (Bitsios et al., 2004).

Affective evaluations of stimuli. Before the start of the training procedure, and after each scanning session, we asked subjects to rate the pleasantness of the shape images and the liquid outcomes. Within each category, stimuli were presented in random order and subjects reported their evaluation by moving a cursor along a scale from -5 to +5 .

Swallowing motion. A motion-sensitive inductive coil was positioned on top of the subjects' throat using a Velcro strap around the neck. This measured the motion of the subjects' throat during swallowing. The time course derived from this measure was used as a regressor of no interest in the fMRI data analysis. We do not have recordings for one subject who found the coil uncomfortable.

\section{fMRI scanning procedure}

fMRI data were acquired on a Siemens 3T TRIO MRI scanner; BOLD contrast was measured with gradient echo $\mathrm{T}^{*}$-weighted echo-planar images (EPIs). Imaging parameters were optimized to minimize signal dropout in medial ventral prefrontal and anterior ventral striatum: we used a tilted acquisition sequence at $30^{\circ}$ to the anterior cingulateposterior cingulate line (Deichmann et al., 2003), and an eight-channel phased array coil, which yields a $\sim 40 \%$ signal increase in this area over a standard coil. The first five volumes of each session were discarded to permit T1 equilibration. Other parameters were as follows: 36 slices, in-plane resolution, $3 \times 3 \mathrm{~mm}$; slice thickness, $3 \mathrm{~mm}$; repetition time, $2.25 \mathrm{~s}$; echo time, $30 \mathrm{~ms}$; field of view, $192 \times 192 \mathrm{~mm}$. A T1-weighted structural image was also acquired for each subject, as well as a 49 -slice EPI to improve coregistration.

\section{Imaging data processing and analysis}

Data were preprocessed using the SPM5 software package (http://www. fil.ion.ucl.ac.uk/spm/software/spm5/). Images were corrected for slice timing and spatially realigned to the first image from the first session. One of the 49-slice EPIs collected at the end of the experiment was used to improve coregistration and spatial normalization. The 36-slice EPIs were coregistered to a 49-slice EPI, which was in turn coregistered to the T1-weighted anatomical scan. The T1 image was segmented into white and gray matter, and the gray matter was coregistered and normalized to the template gray matter image distributed with SPM5 (in Montreal
Neurological Institute space). These parameters were subsequently applied to the T1 image itself as well as the set of 36-slice EPIs. Spatial smoothing was then applied to the 36-slice EPIs using a Gaussian kernel with a full width at half maximum of $8 \mathrm{~mm}$.

Statistical analysis was performed using a general linear model (GLM). The transfer session was modeled separately from the three training sessions, and here we report results only from the transfer phase of the experiment. The GLM included regressors at the time of cue onset for five conditions: specific transfer when the option compatible with the pavlovian cue was chosen, specific transfer when the incompatible option was chosen, pavlovian reward control, pavlovian neutral control, and neutral choice control. We also included regressors at the time of expected outcome. Each regressor was modeled as an impulse function $(0 \mathrm{~s})$, and convolved with the canonical hemodynamic response function. Regressors of no interest included missed trials when no option was chosen, the six ongoing motion parameters estimated during realignment, and motion caused by swallowing. The results from each subject were taken to the random effects level by applying $t$ tests between contrast images to produce group statistical parametric maps.

\section{Results}

\section{Behavioral results}

Results of pavlovian training

Behavioral results indicate that the pavlovian stimulus-outcome associations were successfully learned. After each training session, subjects were asked to rate on a scale from -5 to +5 how pleasant they found each shape stimulus and each liquid. After training, subjects rated the stimuli associated with rewarding outcomes as significantly more pleasant than the stimulus associated with the neutral outcome (paired $t$ test, $t_{(22)}=-3.0840$; $p<$ 0.01 ) (Fig. 2a). Pupil reflex amplitude also discriminated between reward and neutral conditions (Fig. $2 b$ ). In the 16 subjects who showed reliable amplitude changes in pupil diameter after cue presentation, the peak amplitude is significantly smaller for rewarded outcome trials, which indicates a higher degree of arousal when subjects saw reward predictive cues (paired $t$ test, $t_{(15)}=2.4173 ; p<0.05$ ) (Bitsios et al., 2004; Seymour et al., 2007).

\section{Initial learning of instrumental associations}

Subjects' choice behavior in the instrumental trials indicated that the instrumental associations were acquired. During the final training session subjects were significantly more likely to choose the action delivering a reward outcome when the alternative action delivered the neutral solution (Fig. 2c) (one-sided paired $t$ test, $\left.t_{(22)}=1.8399 ; p<0.05\right)$.

\section{Outcome-selective transfer effects during test phase}

We found evidence for an outcome-specific transfer effect in subjects' choice behavior during the transfer test phase. During the transfer phase, subjects choose the compatible option on average $66 \%$ of the time; this is significantly higher than cue invariant responding which averages to $50 \%$ over the two outcome-specific conditions (paired $t$ test, $t_{(22)}=3.6348 ; p<0.005$ ). There were a total of 50 specific transfer trials for each subject and, separating these into five 10-trial bins, we found that there was neither a significant increase or decrease in choice allocation across time 
a
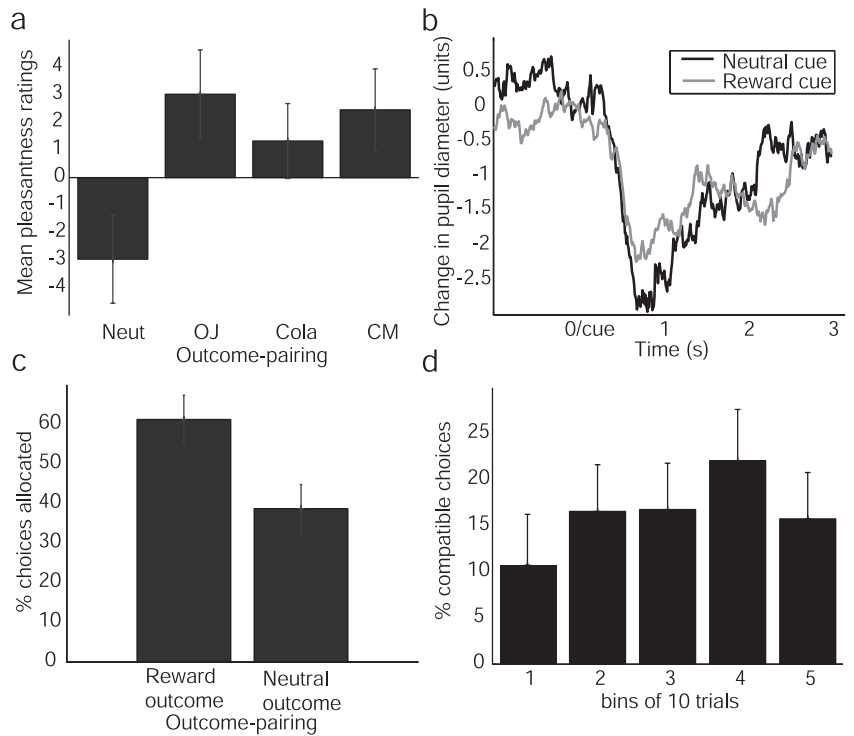

Figure 2. Behavior during training and test sessions. $\boldsymbol{a}$, Mean pleasantness ratings for visual cue stimuli after the training sessions, plotted by outcome pairing. The cues paired with the neutral outcome were rated as significantly less pleasant than the cues paired with reward outcomes (paired $t$ test, $t_{(22)}=-3.0840 ; p<0.01$ ). $\boldsymbol{b}$, Pupil diameter in response to visual cues. The peak amplitude is significantly smaller for the cues paired with reward outcomes for the 16 subjects who showed reliable amplitude changes after cue presentation (paired $t$ test, $\left.t_{(15)}=2.4173 ; p<0.05\right)$. c, Choice behavior during the second session of instrumental trials, above cue invariant responding (50\%). Plotted are responses during trials in which subjects chose between a reward outcome and the neutral outcome. Subjects were significantly more likely to choose the action leading to the reward outcome (one-sided paired $t$ test, $t_{(22)}=$ $1.8399 ; p<0.05)$. $\boldsymbol{d}$, Choice data binned into five 10 -trial bins. There is no significant linear trend across the session (linear regression of the percentage of compatible choice allocation onto bin number, $p=0.239$ ). Error bars indicate SEM.

(Fig. $2 d$ ), indicating that the biasing effect of the pavlovian cues on choice persisted for the duration of the extinction test and did not attenuate.

\section{fMRI results}

To gain insight into the mechanisms underlying outcomespecific transfer in humans, we performed two analyses. First, we compared brain activity during trials assessing outcome-specific transfer when subjects chose the option compatible with the pavlovian cue to trials when they chose the incompatible option (one subject who never chose the incompatible cue was excluded from this analysis) (Fig. 3a). We found significant activation in the right ventrolateral putamen $\left(t_{(21)}=3.79, p<0.001\right.$ uncorrected; $x=27, y=-3, z=-3$ ) extending posteriorly toward the palli$\operatorname{dum}\left(t_{(21)}=3.81, p<0.001\right.$ uncorrected; $x=24, y=-18, z=$ $0)$. The left pallidum also showed a peak at this threshold $\left(t_{(21)}=\right.$ $3.82, p<0.001$ uncorrected; $x=-27, y=-15, z=-3)$. These were the only regions to meet our significance criterion in this contrast.

Second, we plotted the average parameter estimates taken from the general linear model estimates at the peak putamen voxel for each subject (Fig. $3 b$ ). We found that the difference between conditions was caused by a significant decrease in signal during the outcome-specific trials where the incompatible response was chosen, relative to the outcome-specific trials when the compatible response was chosen and to the other control conditions. In fact, activity in the compatible condition did not differ significantly from activity during any of the other control conditions (paired $t$ tests, $p>0.05$ ) and, more generally, activity a

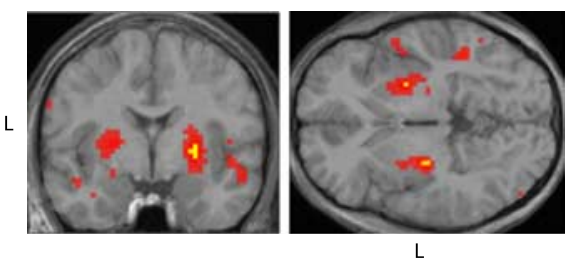

b

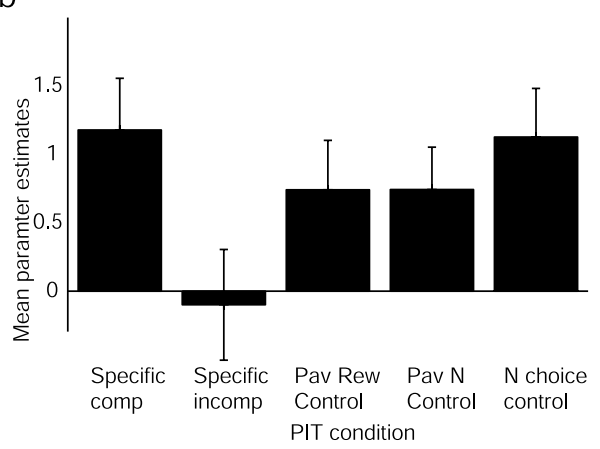

Figure 3. Imaging results from the pavlovian-instrumental transfer (PIT) phase. $\boldsymbol{a}$, fMRI results from the contrast comparing the outcome-specific transfer trials in which the action compatible with the pavlovian cue is selected to those in which the incompatible action is selected (red, $p<0.01$; yellow, $p<0.001$ ). At a threshold of $p<0.001$, uncorrected, we find significant activation in the ventrolateral putamen $\left(t_{(21)}=3.79 ; p<0.001\right.$, uncorrected; $x=$ $27, y=-3, z=-3)$ and bilateral pallidum $\left(t_{(21)}=3.81, p<0.001\right.$, uncorrected, $x=24$, $y=-18, z=0 ;$ and $t_{(21)}=3.82, p<0.001$, uncorrected, $\left.x=-27, y=-15, z=-3\right) \cdot \boldsymbol{b}$, Parameter estimates from the peak putamen voxel for each subject for each of the five experimental conditions during the transfer phase [specific compatible (comp), specific incompatible (incomp), pavlovian reward (pav rew) control, pavlovian neutral (pav N) control, and neutral (N) choice control]. Parameter estimates in the specific compatible condition do not differ significantly from any condition other than specific incompatible (paired $t$ tests, $p>0.05$ ). Error bars indicate SEM.

in the outcome-specific trials did not differ from the control conditions (paired $t$ tests, $p>0.05$ ).

\section{Discussion}

Our results provide insights into the neural mechanisms by which pavlovian cues can modulate choice between different instrumental courses of action, in humans. In outcome-specific transfer, subjects are more likely to choose an action that is associated with a particular outcome in the presence of a pavlovian cue that was previously associated with the presence of that outcome. We found neural correlates of outcome-specific transfer in a very circumscribed region of extended ventral striatum in the ventral caudolateral putamen. This region and an adjacent region of ventral pallidum were the only areas to meet our statistical criterion for significance.

These findings add to an accumulating body of evidence from human fMRI studies of a role for an extended region of ventral parts of putamen alongside the nucleus accumbens in functions related to reward-learning and prediction errors (O'Doherty et al., 2002, 2003; McClure et al., 2003) and now in interactions between pavlovian and instrumental conditioning. Such findings resonate with anatomical and histochemical studies in primates that indicate that ventral parts of putamen share many of the cytoarchitectonic characteristics of nucleus accumbens, as well as sharing similar inputs (Russchen et al., 1985; Selemon and Goldmanrakic, 1985; Fudge and Haber, 2002; Fudge et al., 2002).

The present findings do suggest, however, that different parts of the ventral striatum may contribute differentially to distinct forms of pavlovian-instrumental transfer in humans. This sug- 
gestion is based on a comparison of our finding that the ventrolateral putamen is involved in outcome-specific transfer in humans with the results of a previous study implicating nucleus accumbens in the general excitatory effects of pavlovian cues on instrumental performance (Talmi et al., 2008). It is well established that pavlovian cues can exert a general, nonspecific excitatory effect on the performance of instrumental actions (Estes, 1943, 1948; Rescorla and Solomon, 1967; Colwill and Rescorla, 1988; Rescorla, 1994; Holland, 2004), an effect that Talmi et al. (2008) demonstrated is mediated by activation of nucleus accumbens and of amygdala. In the context of the present study, these findings suggest that outcome-specific and general transfer may depend on quite distinct neural substrates in humans, mirroring clear double dissociations between the neural circuits known to be involved in implementing these effects in rodents (Corbit et al., 2001; Corbit and Balleine, 2005). Although the present study was not designed to assess the effects of general transfer, in future it will be important to compare and contrast outcome-specific and general transfer effects within the same fMRI study to provide a more direct test of the hypothesis that, as in rodents, outcome-specific and general transfer in humans depend on distinct components of the ventral striatum.

Note that although we found a remarkably good correspondence between our findings and those from the rodent lesion studies at the level of the ventral striatum, other regions in addition to the ventral striatum have been implicated in specific pavlovian-instrumental transfer in rodents, including the basolateral amygdala (Corbit and Balleine, 2005) and dorsolateral striatum (Corbit and Janak, 2007). We did not find any evidence for a differential contribution of these regions in the present study. One possibility is that these areas do play a role in specific transfer effects in humans, but this does not result in a global increase in activity between conditions and, thus, does not become manifest with BOLD fMRI.

The present results go beyond merely pointing to homologies between outcome-specific transfer effects in rodents and humans. Previous animal studies on this topic have all involved lesion manipulations, which, although important for identifying whether a given region is necessary for implementing specific transfer effects, cannot provide insight into the neural computations underlying such an effect. Here, we measured dynamic changes in BOLD responses as subjects made choices that were either consistent or inconsistent with the specific transfer effect. Responses consistent with the specific transfer effect occurred when subjects chose the outcome compatible with the pavlovian cue, and inconsistent responses occurred when subjects chose the incompatible option. Although subjects showed a significant bias toward the compatible action overall, sometimes they chose the incompatible action; this allowed us to compare activity when transfer guided behavior with activity under identical stimulus conditions when subjects chose independently of the cue. Activity in ventrolateral putamen was not significantly elevated on trials when an outcome-specific cue was presented compared with control trials where cues for other, unavailable outcomes were presented, suggesting that outcome-specific transfer effects are not mediated by an overall increase in activity in this area. Furthermore, even on outcome-specific trials where subjects chose the action compatible with the pavlovian cue, there was no increase in activity compared with non-outcome-specific control trials. Instead, we found a significant decrease in signal on those outcome-specific trials where subjects chose the action incompatible with the outcome, compared with compatible choice outcome-specific trials.
This finding provides insight into the computations that might be taking place in the ventral striatum during outcomespecific transfer effects. Outcome-specific transfer effects are thought to be mediated by outcome-response (O-R) associations that are activated by the pavlovian cues (Rescorla, 1994; Balleine and Ostlund, 2007). A natural hypothesis is that when the action plan activated by the $\mathrm{O}-\mathrm{R}$ association is feasible (because such an action is available), it must be inhibited before another action can be taken. Note that, under this hypothesis, the $\mathrm{O}-\mathrm{R}$ association needs to be inhibited during the outcomespecific transfer trials when the incompatible response is chosen, but not when the compatible response is selected, or in any of the other control trials. This provides a computational explanation for why suppression of activity in the ventrolateral putamen is observed only in the incompatible outcome-specific transfer trials.

Specific transfer effects from pavlovian cues have been argued to play a role in addictive behaviors (Ludwig et al., 1974). For example, Hogarth et al. (2007) demonstrated specific transfer of a tobacco-seeking response in the presence of a tobacco predicting cue, relative to a money predicting cue. Here, we demonstrate similar behavioral results, using nonaddictive outcomes, indicating that the observed transfer effects reflect a general property of reward-associated cues that are not specifically related to addictive stimuli. Nonetheless, there are clear parallels between our experimental design and the potential influence of environmental cues on drug-seeking behavior. Our fMRI results suggest the hypothesis that suppression of an outcome-response association might contribute toward biasing behavior away from cuecompatible responding. This raises the possibility of a future therapeutic intervention in addiction, in which ventrolateral putamen circuitry could potentially be targeted (for instance via a neurofeedback procedure) (deCharms et al., 2005; Bray et al., 2007) to suppress effects of environmental drug cues on drugseeking behavior.

In this study, we demonstrated an outcome-specific pavlovian-instrumental transfer effect in humans, which serves to bias action choice toward actions associated with an outcome consistent with a concurrently presented cue. BOLD fMRI measured while subjects performed this task demonstrated a signal decrease in ventrolateral putamen when subjects' chose the action incompatible with the cue. This finding points to a computational role for this region in suppressing outcome-response associations, necessary to perform an action incompatible with the pavlovian cue only when a compatible action is feasible. This work adds to our understanding of the neural mechanisms of stimulus-outcome guided decision making in both animals and humans, which is fundamental for understanding maladaptive choice behaviors such as addiction.

\section{References}

Balleine B (1994) Asymmetrical interactions between thirst and hunger in pavlovian-instrumental transfer. Quart J Exp Psychol B 47:211-231.

Balleine BW, Ostlund SB (2007) Still at the choice-point: action selection and initiation in instrumental conditioning. Ann NY Acad Sci 1104:147-171.

Bitsios P, Szabadi E, Bradshaw CM (2004) The fear-inhibited light reflex: importance of the anticipation of an aversive event. Int J Psychophysiol 52:87-95.

Blundell P, Hall G, Killcross S (2001) Lesions of the basolateral amygdala disrupt selective aspects of reinforcer representation in rats. J Neurosci 21:9018-9026.

Bray S, Shimojo S, O’Doherty JP (2007) Direct instrumental conditioning of neural activity using functional magnetic resonance imaging-derived reward feedback. J Neurosci 27:7498-7507. 
Colwill RM, Rescorla RA (1988) Associations between the discriminative stimulus and the reinforcer in instrumental learning. J Exp Psychol Anim Behav Process 14:155-164.

Corbit LH, Balleine BW (2005) Double dissociation of basolateral and central amygdala lesions on the general and outcome-specific forms of pavlovian-instrumental transfer. J Neurosci 25:962-970.

Corbit LH, Janak PH (2007) Inactivation of the lateral but not medial dorsal striatum eliminates the excitatory impact of pavlovian stimuli on instrumental responding. J Neurosci 27:13977-13981.

Corbit LH, Muir JL, Balleine BW (2001) The role of the nucleus accumbens in instrumental conditioning: evidence of a functional dissociation between accumbens core and shell. J Neurosci 21:3251-3260.

deCharms RC, Maeda F, Glover GH, Ludlow D, Pauly JM, Soneji D, Gabrieli JD, Mackey SC (2005) Control over brain activation and pain learned by using real-time functional MRI. Proc Natl Acad Sci USA 102:18626-18631.

Deichmann R, Gottfried JA, Hutton C, Turner R (2003) Optimized EPI for fMRI studies of the orbitofrontal cortex. NeuroImage 19:430-441.

Estes WK (1943) Discriminative conditioning. I. A discriminative property of conditioned anticipation. J Exp Psychol 32:150-155.

Estes WK (1948) Discriminative conditioning. 2. Effects of a pavlovian conditioned stimulus upon a subsequently established operant response. J Exp Psychol 38:173-177.

Fudge JL, Haber SN (2002) Defining the caudal ventral striatum in primates: cellular and histochemical features. J Neurosci 22:10078-10082.

Fudge JL, Kunishio K, Walsh P, Richard C, Haber SN (2002) Amygdaloid projections to ventromedial striatal subterritories in the primate. Neuroscience 110:257-275.

Hogarth L, Dickinson A, Wright A, Kouvaraki M, Duka T (2007) The role of drug expectancy in the control of human drug seeking. J Exp Psychol Anim Behav Process 33:484-496.

Holland PC (2004) Relations between pavlovian-instrumental transfer and reinforcer devaluation. J Exp Psychol Anim Behav Process 30:104-117.
Ludwig AM, Wikler A, Stark LH (1974) First drink: psychobiological aspects of craving. Arch Gen Psychiatry 30:539-547.

McClure SM, Berns GS, Montague PR (2003) Temporal prediction errors in a passive learning task activate human striatum. Neuron 38:339-346.

O’Doherty JP, Deichmann R, Critchley HD, Dolan RJ (2002) Neural responses during anticipation of a primary taste reward. Neuron 33:815-826.

O’Doherty JP, Dayan P, Friston K, Critchley H, Dolan RJ (2003) Temporal difference models and reward-related learning in the human brain. Neuron 38:329-337.

O’Doherty JP, Buchanan TW, Seymour B, Dolan RJ (2006) Predictive neural coding of reward preference involves dissociable responses in human ventral midbrain and ventral striatum. Neuron 49:157-166.

Ostlund SB, Balleine BW (2007) Selective reinstatement of instrumental performance depends on the discriminative stimulus properties of the mediating outcome. Learn Behav 35:43-52.

Rescorla RA (1994) Transfer of instrumental control mediated by a devalued outcome. Anim Learn Behav 22:27-33.

Rescorla RA, Solomon RL (1967) Two-process learning theory: relationships between pavlovian conditioning and instrumental learning. Psycho Rev 74:151-182.

Russchen FT, Bakst I, Amaral DG, Price JL (1985) The amygdalostriatal projections in the monkey. An anterograde tracing study. Brain Res 329:241-257.

Selemon LD, Goldmanrakic PS (1985) Longitudinal topography and interdigitation of corticostriatal projections in the rhesus monkey. J Neurosci 5:776-794.

Seymour B, Daw N, Dayan P, Singer T, Dolan R (2007) Differential encoding of losses and gains in the human striatum. J Neurosci 27:4826-4831.

Smeets PM, Barnes-Holmes D (2003) Children's emergent preferences for soft drinks: stimulus-equivalence and transfer. J Econ Psychol 24:603-618.

Talmi D, Seymour B, Dayan P, Dolan RJ (2008) Human pavlovianinstrumental transfer. J Neurosci 28:360-368. 